\title{
Accurate Modelling of Hollow Core Photonic Bandgap Fibre
}

\author{
Eric Numkam Fokoua, Seyed Reza Sandoghchi, Yong Chen, Natalie. V. Wheeler, Naveen K. Baddela, \\ John R. Hayes, Marco N. Petrovich, David J. Richardson and Francesco Poletti \\ Optoelectronics Research Centre, University of Southampton, Highfield Campus, SO17 1BJ Southampton, UK \\ ernf1g10@orc.soton.ac.uk
}

\begin{abstract}
A modelling tool to accurately reproduce the performance of fabricated hollow-core photonic bandgap fibers from their SEM images is presented. This enables new understanding of the effect of cross-sectional distortions.

OCIS codes: (060.4005) Microstructured fibers; (060.2280) Fiber design and fabrication
\end{abstract}

Hollow core photonics bandgap fibres (HC-PBGFs) open unprecedented application opportunities in areas that include data transmission with low latency, ultralow nonlinearity and improved robustness to ionizing radiation, mid-IR gas spectroscopy and high power laser delivery [1]. To improve the optical performance of HC-PBGFs further (e.g. reduce their loss, widen their transmission bandwidth, control their dispersive and modal properties), it is critical to be able to understand and control the effect of various types of small scale distortions that are often present in their cross section. Conventional structural reconstruction methods based on edge-detection routines are unable to capture the finest details with the accuracy required for this task [2,3]. Recently we have proposed a novel method to capture the structural irregularities of fabricated fibres with the nanometer scale accuracy required [4]. Using image processing tools we automatically detect the hole and rod positions, which are then used to build a reconstructed fibre cross section using information on average membrane thickness and rod sizes and imposing mass conservation arguments.

Here we apply this new method to the study of several fabricated HC-PBGFs and we demonstrate that for the first time it is possible to simulate with high accuracy and with no free parameters not only the scattering and absorption loss of these fibres, but also their optical bandwidth and, even more strikingly, to accurately match the presence or absence of surface modes (Fig.1A). The accurate reproduction of fabricated HC-PBGF cross-sections allows us to study the dispersive properties of all their guided modes (Fig.1B), their differential loss, and more in general to understand which structural features are responsible for the guidance of surface modes and/or for an excess loss in the fibers. We believe that using this understanding it will be possible to improve fabrication processes to realize HC-PBGFs with greatly improved performance.
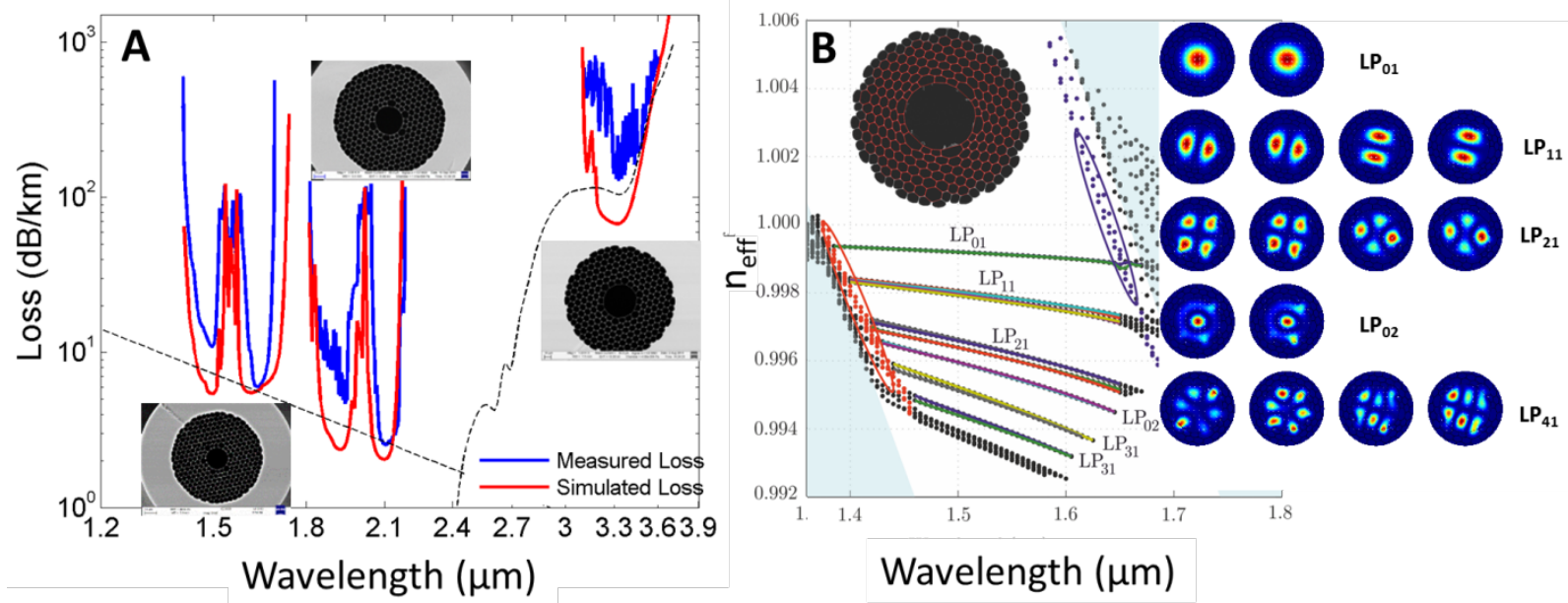

Fig. 1: (A) Simulated and measured loss of 3 HC-PBGFs guiding at 1550, $2000 \mathrm{~nm}$ (surface scattering loss dominated) and at $3300 \mathrm{~nm}$ (glass absorption dominated). (B) simulated modal dispersion and modal intensities for a fabricated wide bandwidth HC-PBGF.

\section{References}

[1] Poletti et al. Nanophotonics, 2, 315-340 (2013).

[2] Li et al, J. Lightwave Technol. 25, 2463-2468 (2007).

[3] Aghaie et al, J. Lightwave Technol. 31, 1015-1022 (2013).

[4] Numkam et al. in proc. OFC 2014, paper M2F.5

This work was supported by the EU 7th Framework Programme under grant agreement 258033(MODE-GAP) and by the Royal Society (UK) 